\title{
Fracture criterion based on the higher-order asymptotic fields
}

\author{
YUEGUANG WEI and TZUCHIANG WANG \\ LNM, Institute of Mechanics, Academia Sinica, Beijing 100080, People's Republic of China
}

Received 21 December 1993; accepted 21 May 1995

\begin{abstract}
A complete development for the higher-order asymptotic solutions of the crack tip fields and finite element calculations for mode I loading of hardening materials in plane strain are performed. The results show that in the higher-order asymptotic solution (to the twentieth order), only three coefficients are independent. These coefficients are determined by matching with the finite element solutions carried out in the present paper (our attention is focused on the first five terms of the higher-order asymptotic solution). We obtain an analytic characterization of crack tip fields, which conform very well to the finite element solutions over wide range.

A modified two parameter criterion based on the asymptotic solution of five terms is presented. The upper bound and lower bound fracture toughness curves predicted by modified two parameter criterion are given. These two curves agree with most of the experimental data and fully capture the proper trend.
\end{abstract}

\section{Introduction}

The development of the HRR solution by Hutchinson [1] and Rice and Rosengren [2] for planar crack problems is a milestone in the area of elastic-plastic fracture mechanics, demonstrating that the strength of the singularity in a power law hardening material is uniquely determined by the $J$-integral defined by Rice [3]. Since that time, using these theoretical developments as a foundation, much work has been done to determine the conditions under which the near tip fields will be dominated by the HRR singularity field. Results show that the HRR singularity field can characterize the high triaxiality elastic plastic field near the crack tip, but for the low triaxiality case, the situation will be different. High triaxiality is only one of many possible states. Even though a crack tip field could be of a high triaxiality state during the initial loading stage, it will be changed gradually into low triaxiality as load increases from small scale yielding to large scale yielding, and the HRR solution will deviate from the finite element solution (O'Dowd and Shih [4]). The cases of a single-edge shallow crack (Ai-Ani and Hancock [5]), center cracked panel and double-edge cracked panel etc. (O'Dowd and Shih [4]) are examples of the low triaxiality states. In order to develop the more effective elasticplastic fracture criterion, two parameter approaches were developed. $\mathrm{Li}$ and Wang [6] first derived the second order asymptotic field, in which the first term was the HRR singular field, the coefficient of the second term was determined by matching the asymptotic solution with the finite element full field solution. The second order asymptotic solution obtained in such a way was a great improvement on the HRR solution. Sharma and Aravas [7] also completed the second order analysis taking account of possible elasticity effects. Recently, Yang et al. [8] carried out a second-order asymptotic analysis for mode I and mode II cracks in detail, and published the exponent values of higher-order terms. They utilized the two term expansion solution to establish the size and shape of the zone dominated by the HRR field. Xia et al. [9] derived five terms of the asymptotic field for $n=3$ and four terms of an asymptotic field 
for $n=5,7,10$. Five (or four) terms of asymptotic series for stresses had three independent coefficients, and appropriate choices of the latter two coefficients (the first coefficient can be expressed by $J$-integral) could reproduce near tip fields representative of a broad range of crack tip constraints in moderate and low hardening materials. O'Dowd and Shih [10,11] developed a two term solution in which the first term was also the HRR singularity field, and the second term, including function form and its coefficient, was determined by matching this two term solution with the finite element full field solution. They developed a $J-Q$ two parameter criterion dominating crack initiation $(Q$ characterizing stress triaxiality ahead of crack tip). Betegon and Hancock [12] and Ai-Ani and Hancock [5] presented $J-T$ two parameter characterization by carrying out finite element calculations for weakly hardening materials. Here, $T$ was the uniform tension stress parallel to the crack faces and associated with the second term of Williams' expansion.

In this paper, we study the elastic-plastic fracture criterion strictly based on the higherorder asymptotic field. Our attention is focused on characterizing the elastic-plastic field near the crack tip and developing a modified two parameter criterion. Dominating parameters are selected from the coefficients of the asymptotic solution. The evolution of parameters with loading are determined by matching higher-order asymptotic solution with finite element solutions during various loading stages. For this purpose, we carry out the finite element calculations for two representative cases which are the bend cracked panel (BCP) and the center cracked panel (CCP). Furthermore, the fracture toughness curve is predicted and compared to that from the experiment.

\section{The higher-order asymptotic solution}

A widely used uniaxial stress-strain relation is the Ramberg-Osgood form

$$
\frac{\varepsilon}{\varepsilon_{0}}=\frac{\sigma}{\sigma_{0}}+\alpha\left(\frac{\sigma}{\sigma_{0}}\right)^{n}
$$

where $\sigma_{0}$ is an effective yield stress, $\varepsilon_{0}=\sigma_{0} / E$ is the associated elastic strain with $E$ as Young's modulus, $\alpha$ and $n$ are parameters chosen to fit experimental data. $n$ is the strain hardening exponent.

Generalizing (1) to multiaxial states by $J_{2}$ deformational plasticity theory, one obtains the stress strain relation

$$
\varepsilon_{i j}=\frac{1+\nu}{E} s_{i j}+\frac{1-2 \nu}{3 E} \sigma_{k k} \delta_{i j}+\frac{3}{2} \alpha \varepsilon_{0}\left(\frac{\sigma_{e}}{\sigma_{0}}\right)^{n-1} \frac{s_{i j}}{\sigma_{0}}
$$

in which, $s_{i j}$ is the deviatoric stress, $\sigma_{e}=\sqrt{3 s_{i j} s_{i j} / 2}$ is the effective stress, $\nu$ is Poisson's ratio, $i, j=1,3$.

For the plane strain case, (2) can be further simplified, and equilibrium relations can be expressed by a stress function $\phi$ in cylindrical coordinates $(r, \theta)$ as the following

$$
\sigma_{r}=\frac{1}{r}\left(\frac{\partial \phi}{\partial r}+\frac{\partial^{2} \phi}{r \partial \theta^{2}}\right), \sigma_{\theta}=\frac{\partial^{2} \phi}{\partial r^{2}}, \sigma_{r \theta}=-\frac{\partial}{\partial r}\left(\frac{\partial \phi}{r \partial \theta}\right) .
$$

For the plane strain case, the compatibility equation can be written in strain components with cylindrical coordinates as

$$
\frac{1}{r} \frac{\partial^{2}}{\partial r^{2}}\left(r \varepsilon_{\theta}\right)+\frac{1}{r^{2}} \frac{\partial^{2}}{\partial \theta^{2}} \varepsilon_{r}-\frac{1}{r} \frac{\partial}{\partial r} \varepsilon_{r}-\frac{2}{r^{2}} \frac{\partial^{2}}{\partial r \partial \theta}\left(r \varepsilon_{r \theta}\right)=0 .
$$


Traction free conditions on crack face require

$\phi=\frac{\partial \phi}{\partial \theta}=0$, when $\theta=\pi$.

Symmetry conditions are

$\frac{\partial \phi}{\partial \theta}=\frac{\partial^{3} \phi}{\partial \theta^{3}}=0$, when $\theta=0$.

The form of higher-order asymptotic solution of (2)-(6) is usually taken as

$$
\begin{aligned}
\phi= & \sigma_{0}\left(K_{1} r^{s_{1}+2} \tilde{\phi}_{1}(\theta)+K_{2} r^{s_{2}+2} \tilde{\phi}_{2}(\theta)+K_{3} r^{s_{3}+2} \tilde{\phi}_{3}(\theta)\right. \\
& \left.+K_{4} r^{s_{4}+2} \tilde{\phi}_{4}(\theta)+K_{5} r^{s_{5}+2} \tilde{\phi}_{5}(\theta)\right),
\end{aligned}
$$

where $s_{1}<s_{2}<s_{3}<s_{4}<s_{5}$ are referred to the stress exponents; $K_{i}, \tilde{\phi}_{i}(\theta)(i=1,5)$ are coefficients of the asymptotic solution and the angular distribution functions of the stress function.

Substituting (7) into (3), (2), (4), (5) and (6) in sequence, and by comparison of various order exponents of $r$, we arrived at the nonlinear governing equations and boundary conditions on $\tilde{\phi}_{i}$ for the various (five) order problems.

The first order problem corresponds to solving homogeneous nonlinear governing equations with an independent eigenvalue (singularity exponent $s_{1}$ ). This problem was solved early by Hutchinson [1] and Rice and Rosengren [2]. The solution is well known as the HRR singularity solution.

Both the second order and fifth order $(n=3)$ or fourth order $(n=5,10)$ problems are to solve homogeneous linear equations with an independent eigenvalue ( $s_{2}$ for second order, $s_{4}$ for fourth order $n=5,10$ and $s_{5}$ for fifth order $n=3$ ). The forms of equations and boundary conditions of both order problems are the same. Their solutions correspond to two different eigenvalues $\left(s_{2}<s_{4}\right.$, or $\left.s_{2}<s_{5}\right)$. The amplitudes of the solutions of above problems are unknown.

The other order problems correspond to solving non-homogeneous linear equations with known exponents $\left(s_{3}, s_{5}\right)$. The amplitudes of their solutions depend on that of previous solutions.

Solving these governing equations in sequence, we obtain the results of angular distribution functions. The solving process of higher-order asymptotic fields adopted in the present paper is similar to that of Xia et al. [9]. Readers are referred to that paper for detail.

In the present research, we derive the five terms of the higher-order asymptotic solution for $n=3,5,10$. It is worth pointing out that we can adopt the governing equation and boundary conditions of the second order problem to search for all possible independent eigenvalues. By numerical calculation, we find only three independent eigenvalues within the range $\left(s_{1}, 2\right)$ for $n=3,5,10$, so the large range will cover the first twenty terms of the asymptotic solution. Therefore, we discover immediately that only three coefficients are independent within the first twenty terms of the higher-order asymptotic solution. We also point out that the fifth term of the asymptotic solution is important to the characterization of the crack tip field. The five term asymptotic solutions are shown as follows. 
When $n=3$ :

$$
\begin{aligned}
\frac{\sigma_{i j}}{\sigma_{0}}= & k_{1} \bar{r}^{-0.250} \tilde{\sigma}_{i j 1}(\theta)+k_{2} \bar{r}^{-0.013} \tilde{\sigma}_{i j 2}(\theta)+\left(k_{2}^{2} / k_{1}\right) \bar{r}^{0.224} \tilde{\sigma}_{i j 3}(\theta) \\
& +\left(1 / \alpha k_{1}\right) \bar{r}^{0.250} \tilde{\sigma}_{i j 4}(\theta)+k_{5} \bar{r}^{0.382} \tilde{\sigma}_{i j 5}(\theta) .
\end{aligned}
$$

When $n=5$ :

$$
\begin{aligned}
\frac{\sigma_{i j}}{\sigma_{0}}= & k_{1} \bar{r}^{-0.167} \tilde{\sigma}_{i j 1}(\theta)+k_{2} \bar{r}^{0.054} \tilde{\sigma}_{i j 2}(\theta)+\left(k_{2}^{2} / k_{1}\right) \bar{r}^{0.276} \tilde{\sigma}_{i j 3}(\theta) \\
& +k_{4} \bar{r}^{0.341} \tilde{\sigma}_{i j 4}(\theta)+\left(k_{2}^{3} / k_{1}^{2}\right) \bar{r}^{0.497} \tilde{\sigma}_{i j 5}(\theta) .
\end{aligned}
$$

When $n=10$ :

$$
\begin{aligned}
\frac{\sigma_{i j}}{\sigma_{0}}= & k_{1} \bar{r}^{-0.091} \tilde{\sigma}_{i j 1}(\theta)+k_{2} \bar{r}^{0.070} \tilde{\sigma}_{i j 2}(\theta)+\left(k_{2}^{2} / k_{1}\right) \bar{r}^{0.230} \tilde{\sigma}_{i j 3}(\theta) \\
& +k_{4} \bar{r}^{0.270} \tilde{\sigma}_{i j 4}(\theta)+\left(k_{2}^{3} / k_{1}^{2}\right) \bar{r}^{0.391} \tilde{\sigma}_{i j 5}(\theta),
\end{aligned}
$$

in which $\bar{r}=r /\left(J / \sigma_{0}\right), k_{i}=K_{i}\left(J / \sigma_{0}\right)^{s_{i}}(i=1,2,5$ for $n=3$ and $i=1,2,4$ for $n=5,10)$, three independent coefficients (or parameters) are $J, k_{2}, k_{5}$ for $n=3$ or $J, k_{2}, k_{4}$ for $n=5$ and $n=10, \tilde{\sigma}_{i j}$ are angular distribution functions and their variations are shown in Fig. $1{ }^{1}$ The first term of the asymptotic solution is the HRR singularity field. The $k_{1}$ is formulated with material constants: $k_{1}=1 /\left(\alpha \varepsilon_{0} I_{n}\right)^{\frac{1}{n+1}}$, in which $I_{n}$ is a constant dependent on the $n . I_{n}$ is calculated by the first order angular distribution functions of the stresses and displacements (here, subscribt 1 is neglected):

$$
\begin{aligned}
I_{n}= & \int_{-\pi}^{\pi}\left\{\frac{n}{n+1} \tilde{\sigma}_{e}^{n+1} \cos \theta-\left[\operatorname { s i n } \theta \left(\tilde{\sigma}_{r r}\left(\tilde{u}_{\theta}-\frac{\partial \tilde{u}_{r}}{\partial \theta}\right)\right.\right.\right. \\
& \left.\left.\left.-\tilde{\sigma}_{r \theta}\left(\tilde{u}_{r}+\frac{\partial \tilde{u}_{\theta}}{\partial \theta}\right)\right)+\frac{1}{n+1}\left(\tilde{\sigma}_{r r} \tilde{u}_{r}+\tilde{\sigma}_{r \theta} \tilde{u}_{\theta}\right) \cos \theta\right]\right\} \mathrm{d} \theta .
\end{aligned}
$$

$J$-integral is determined by annulus integration around the crack tip which depends on the external loads.

In later sections, we shall apply higher-order asymptotic field to the characterization of the elastic-plastic field near the crack tip. The other two independent parameters $k_{2}$ and $k_{5}$ ( $n=3)$ or $k_{2}$ and $k_{4}(n=5,10)$ will be determined by matching with the finite element results. Based on the higher-order asymptotic solution, we shall develop an elastic plastic fracture criterion.

\section{Finite element analyses}

In this paper, we develop a two-dimensional elastic-plastic finite element program. This program, based on the $J_{2}$ flow theory of plasticity and adopting the 8-nodal isoparametric element and the tangential stiffness method, was shown to be reliable by calculations of typical examples. Firstly, we calculated the elastic-plastic solution near the crack tip under

${ }^{1}$ Note that the third order stress angular distribution functions $\tilde{\sigma}_{i j 3}(\theta)$ given in [9] is incorrect. Recently, Xia et al. found an error in their program; after rectifying, they obtained a corrected result which is completely in conformity with our result. 

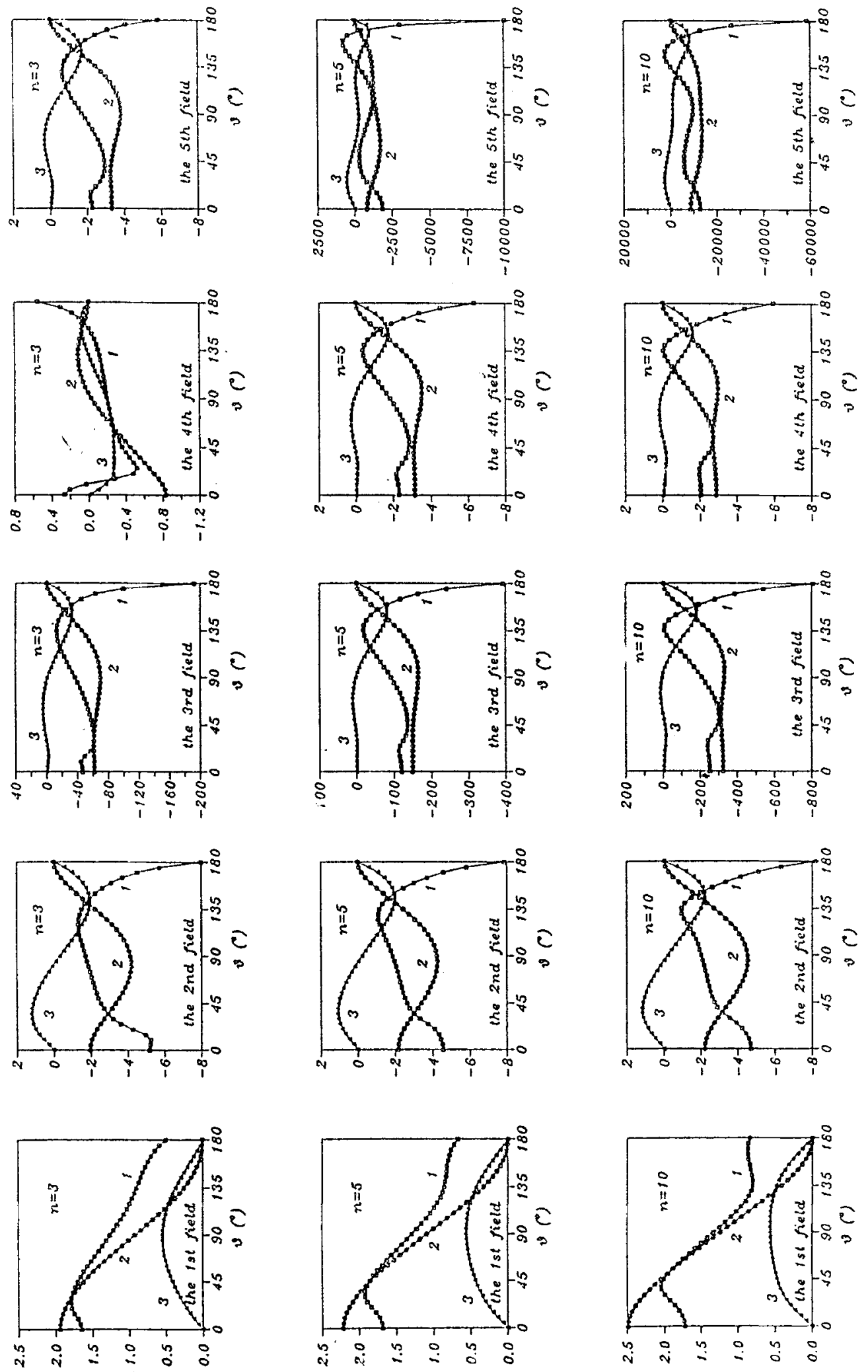

Fig. 1. Angular distributions of the first- to fifth-order stress fields for $n=3,5,10,1$ for $\dot{\sigma}_{r i}, 2$ for $\dot{\sigma}_{\eta_{2}}$ and 3 for $\tilde{\sigma}_{r \vartheta}$. 
small scale yielding and mode I condition. The deviations of $J$-integral values obtained by integrations along the different contours are within one percent. This shows conservation of the $J$-integral. The difference of both $J$-integral values calculated by the contour integration and directly by the external $K$ field is also within one percent. Secondly, we calculated the elastic-plastic fields near the crack tip for single edge cracked panel under tension. The result was in good agreement with that presented by Shih and German [13] for the same problem.

The program is designed according to $J_{2}$ flow theory of plasticity. The incremental relation of multiaxial stress and strain is

$$
d \sigma_{i j}=\frac{E}{1+\nu}\left\{\delta_{i m} \delta_{j n}+\frac{\nu}{1-\nu} \delta_{i j} \delta_{m n}-\frac{9 \mu \omega}{(6 \mu+2 H) \sigma_{e}^{2}} s_{i j} s_{m n}\right\} \mathrm{d} \varepsilon_{m n},
$$

in which $\mu$ is the shear modulus and $H$ is the tangential modulus of plasticity, which by (1) is

$$
H=\frac{d \sigma_{e}}{d \bar{\varepsilon}^{p}}=\frac{\sigma_{0}}{n \alpha \varepsilon_{0}}\left(\frac{\sigma_{e}}{\sigma_{0}}\right)^{1-n}
$$

while parameter $\omega$ is as the following

$$
\omega= \begin{cases}1 & \text { on the loading surface and } s_{i j} d \varepsilon_{i j}>0 \\ 0 & \text { otherwise. }\end{cases}
$$

We carry out the finite element calculations for BCP specimens with $n=5$ and $n=10$ and for CCP specimens with $n=3$ and $n=10$ from small scale yielding to large scale yielding. During the calculations, the ratio of the crack length (a) to the specimen width (W) is taken as 0.5 for various cases, and the other material parameters are taken as $E / \sigma_{0}=500, \nu=$ $0.3, \alpha=1$. The adopted finite element mesh is shown in Fig. 11 .

\section{Characterization of crack tip fields}

First, the finite element results of small scale yielding carried out by O'Dowd and Shih [10] were adopted here to facilitate the comparison with the present higher-order asymptotic solution. Only two coefficients $k_{2}$ and $k_{4}$ need to be determined (here $n=10$ ). In this study, the 'point matching' is enforced at $r /\left(J / \sigma_{0}\right)=2$ and $\theta=0$ for $\left(\sigma_{r r} / \sigma_{0}\right)_{\text {diff }}$ and $\left(\sigma_{\theta \theta} / \sigma_{0}\right)_{\text {diff }}$, in which $(.)_{\text {diff }}$ is the difference field of the finite element result from the HRR solution. Finite element solutions are carried out corresponding to six $T$ stresses (MBL loadings, see [10]). The results for the higher-order asymptotic solution are plotted in Fig. 2. Included in Fig. 3 are the finite element results given in [10]. Comparing Figs. 2 and 3, it is very clear that higher-order asymptotic field characterizes the elastic-plastic fields near the crack tip completely, the full range of difference fields in [10] can be reproduced by the present higher-order asymptotic solutions. The higher-order terms collectively describe a spatially hydrostatic stress of adjustable magnitude.

In the following figures, the comparison of the higher-order asymptotic field with the finite element solution calculated by the present paper is given. Two independent parameters $k_{2}$ and $k_{5}$ (or $k_{4}$ ) are determined by matching the asymptotic solution with the finite element solution for $\sigma_{\theta \theta}$. Here, we take matching points of $\sigma_{\theta \theta}$ for both solutions within the fracture process zone $0.5<r / r_{c}<3.0$. For completing this purpose, we adopt the weighted residual method. 

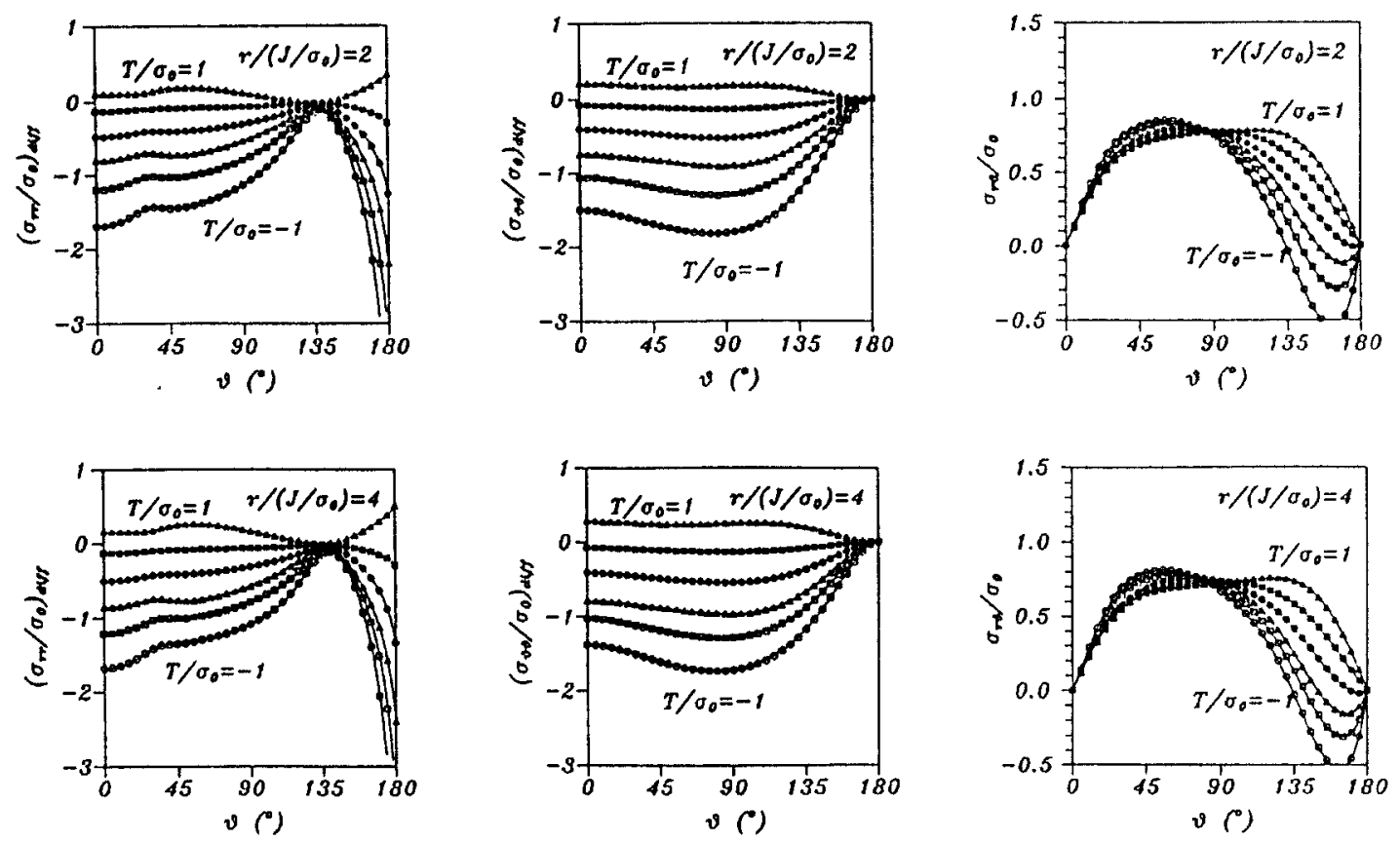

Fig. 2. The sum of the second- to the fifth-order stress fields for $n=10$. Distributions corresponding to six MBL loadings are shown.
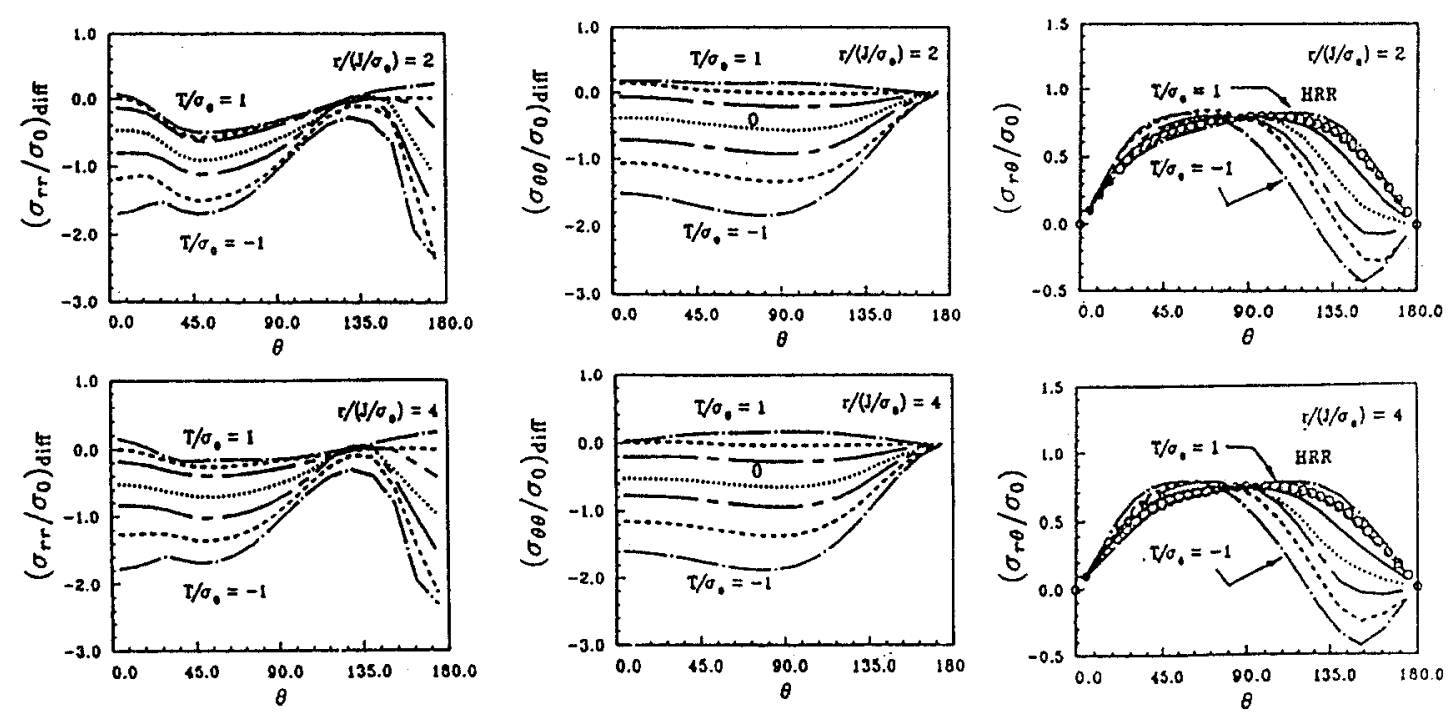

Fig. 3. Difference field from finite element analysis for $n=10$ (O'Dowd and Shih [10]). Distributions corresponding to six MBL loadings are shown.

The parameter $r_{c}$ describes a characteristic scale of the material microstructure. The physical significance of $r_{c}$ was given by Ritchie et al. [14], it will also be introduced in the next section. In the present paper, we take $r_{c} / a$ as 0.01 .

In Fig. 4, the variations of $\sigma_{\theta \theta} / \sigma_{0}$ for three kinds of solutions are shown. From Fig. 4, the HRR singularity field (i.e. first order asymptotic solution) deviates from the finite element solution remarkably. Especially for CCP specimens, the deviation is very large. The higher- 

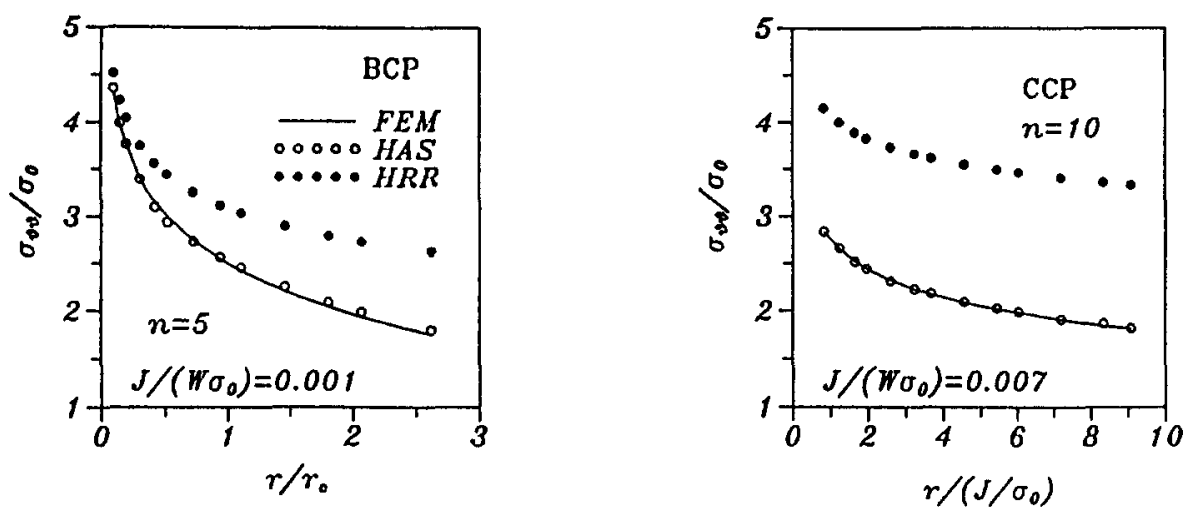

Fig. 4. Stress solutions in three cases for $\mathrm{BCP}$ and $\mathrm{CCP}$ specimens $(\vartheta=0)$.
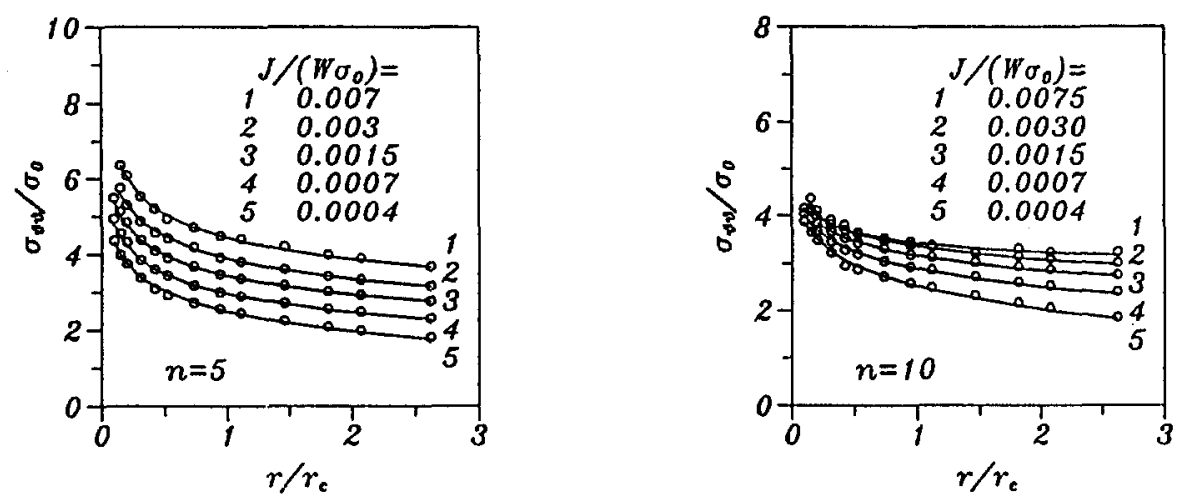

Fig. 5. Comparison of higher-order asymptotic solution (solid line) with finite element solution for BCP specimen $(\theta=0)$.

order asymptotic field of five terms (HAS) is, however, completely consistent with the finite element solution.

In Figs. 5 and 6, the distributions of $\sigma_{\theta \theta} / \sigma_{0}$ along $\theta=0$ ahead of the crack tip for BCP specimens and CCP specimens and for different material hardening exponents under different load levels are given. It is seen from the figures that the higher-order asymptotic solutions conform well to the finite element solutions. The variations of $\sigma_{r r} / \sigma_{0}$ and effective plastic strain $\bar{\varepsilon}^{p} / \alpha \varepsilon$ along $\theta=0$ ahead of the crack tip for BCP specimens and CCP specimens are given in Figs. 7 and 8, respectively. The finite element solutions, the higher-order asymptotic solutions and the HRR solutions are shown simultaneously in three figures. It is clear that the higher-order asymptotic solution is a good simulation for the finite element solution.

Figure 9 shows the variation of coefficients in the higher-order asymptotic field with increase of loading. In Fig. 9, $P$ is the external point load for BCP specimen.

\section{Fracture toughness locus}

Kirk et al. [15] have measured cleavage fracture toughness for A515 steels at room temperature over a broad range of crack tip constraints (the strain hardening exponent $n$ of A515 steel is about 5). They tested edge-cracked bend bars with thicknesses $B=10,25.4$ and $50.8 \mathrm{~mm}$ and various ratios of crack length to width. The measured toughness data $\left(J_{c}\right)$ are related to $Q$. In 

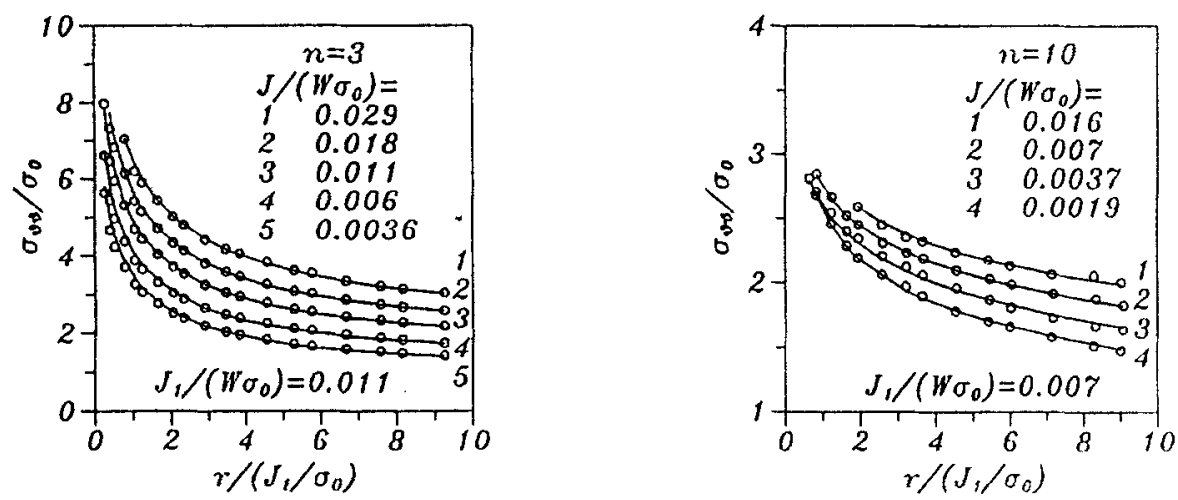

Fig. 6. Comparison of higher-order asymptotic solution (solid line) with the finite element solution for CCP specimen $(\theta=0)$.
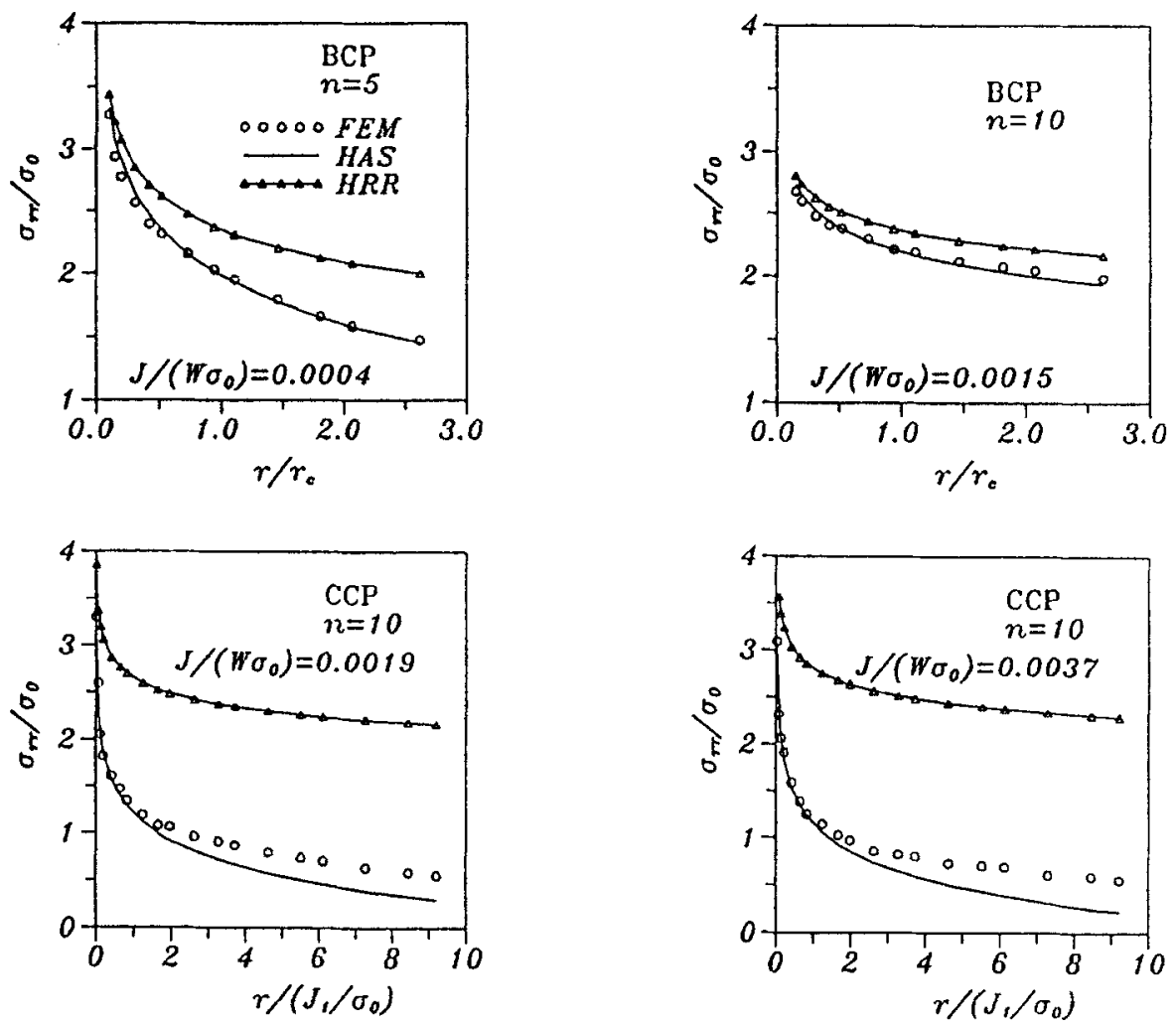

Fig. 7. HRR solution, higher-order asymptotic solution and finite element solution about $\sigma_{r r} / \sigma_{0}$. $\left(\theta=0, J_{1} / W \sigma_{0}=0.007\right)$

here, we can convert the relation $J_{c}-Q$ into $J_{c}-k_{2}$ according to the relation $J_{c}-Q$ from [4]. The experimental data so converted are shown in Fig. 10.

Constraint effects on fracture toughness can be predicted by using the higher-order asymptotic solution in conjunction with a fracture criterion based on the attainment of a critical stress, $\sigma_{\theta \theta}=\sigma_{c}$, at a characteristic microstructural distance, $r=r_{c}$ [14]. Suppose that $r_{c}$ is within the $J-Q$ annulus $\left(1 \leqslant r_{c} \sigma_{0} / J_{c} \leqslant 5\right)$. Now consider the RKR fracture criterion in the higher-order asymptotic field 

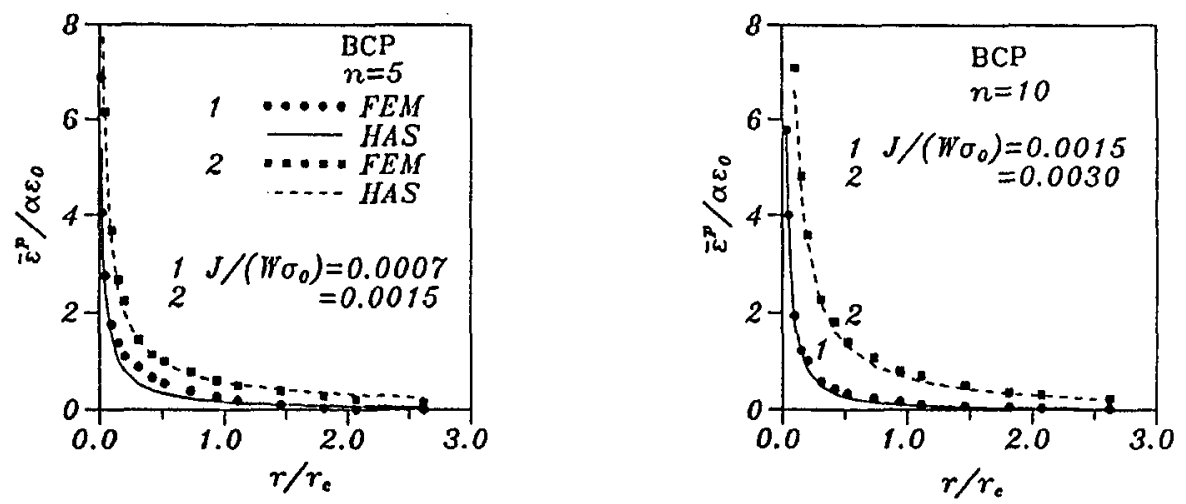

Fig. 8. Distribution of effective plastic strain along $\theta=0$. The comparison of higher-order asymptotic solution with FE solution.
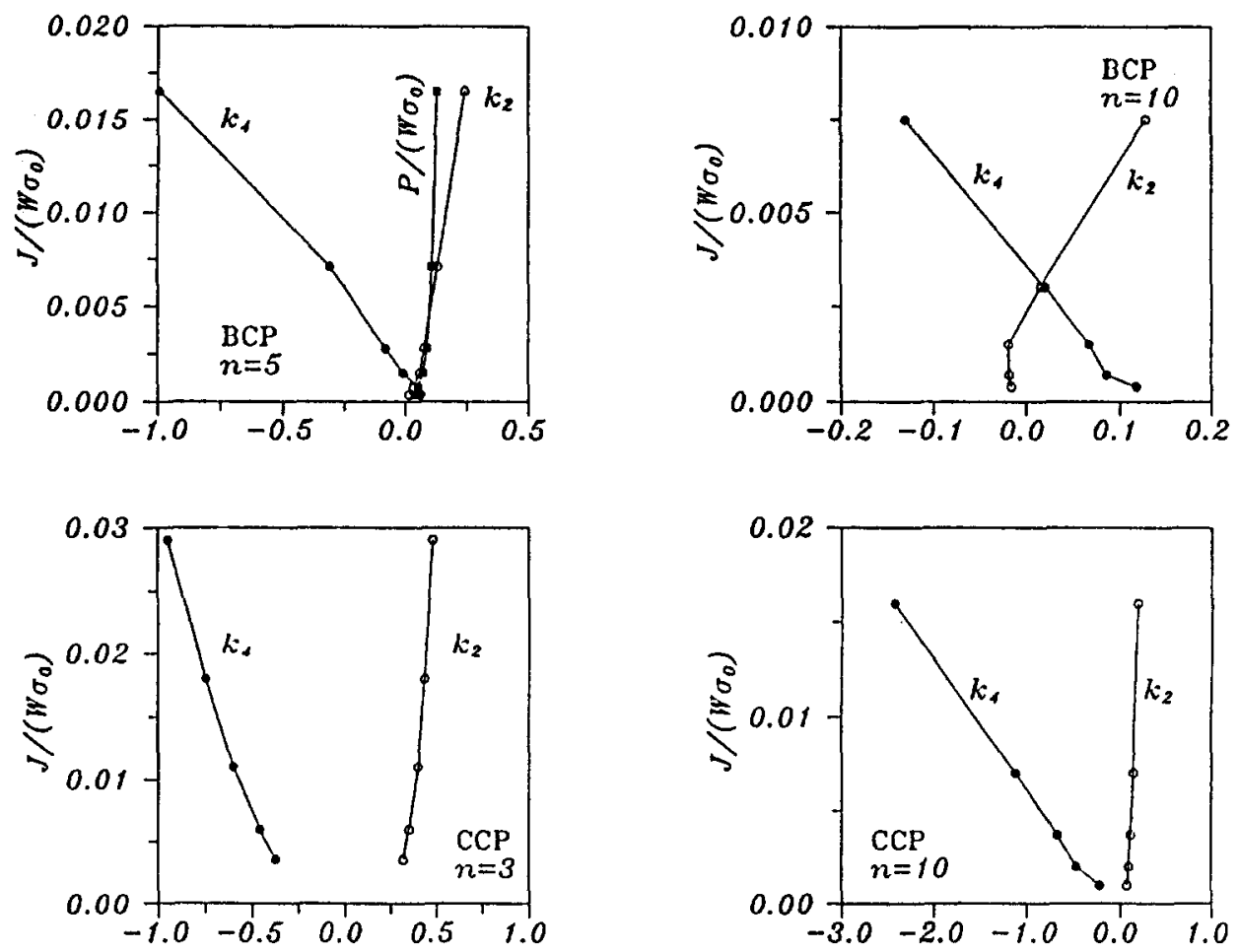

Fig. 9. Parameter-loading relations in higher-order asymptotic solution for $\mathrm{BCP}$ and $\mathrm{CCP}$ specimens.

$$
\begin{aligned}
\frac{\sigma_{c}}{\sigma_{0}}= & k_{1} \bar{r}_{c}^{-0.167} \tilde{\sigma}_{i j 1}(0)+k_{2} \bar{r}_{c}^{0.054} \tilde{\sigma}_{i j 2}(0)+\left(k_{2}^{2} / k_{1}\right) \bar{r}_{c}^{0.276} \tilde{\sigma}_{i j 3}(0) \\
& +k_{4} \bar{r}_{c}^{0.341} \tilde{\sigma}_{i j 4}(0)+\left(k_{2}^{3} / k_{1}^{2}\right) \bar{r}_{c}^{0.497} \tilde{\sigma}_{i j 5}(0)
\end{aligned}
$$

Therefore, we can solve for $J_{c}$ as a function of $k_{2}$ and $k_{4}$ for selected values of $\sigma_{c}$ and $r_{c}$ (noting the relation of $\bar{r}_{c}=r_{c} \sigma_{0} / J_{c}$ ). With $J_{c}^{*}, k_{2}^{*}$ and $k_{4}^{*}$ denoting the corresponding quantities with the remote loading away from the crack tip by $K$ field $(T=0)$, 


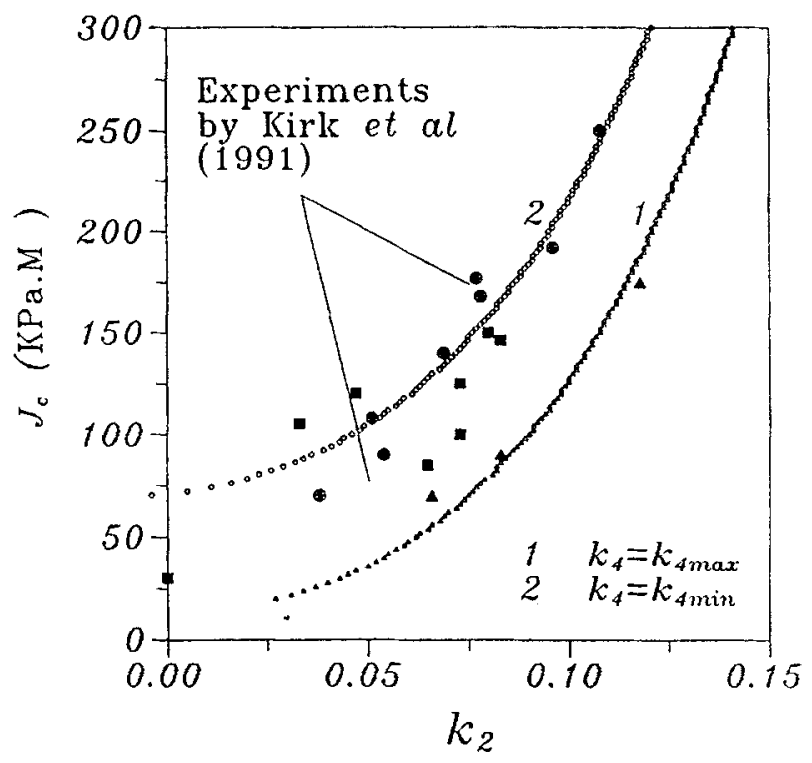

Fig. 10. The comparison of higher order asymptotic solution with experimental results

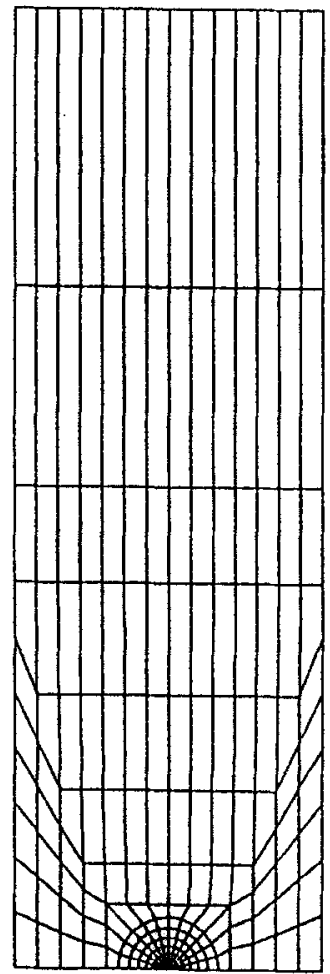

Fig. 11. Finite element mesh.

$$
\frac{J_{c}}{J_{c}^{*}}=\left\{\frac{\sigma_{c} / \sigma_{0}-\Omega\left(k_{2}, k_{4}\right)}{\sigma_{c} / \sigma_{0}}\right\}^{n+1}
$$

in which 


$$
\begin{aligned}
\Omega\left(k_{2}, k_{4}\right)= & k_{2} \bar{r}_{c}^{0.054} \tilde{\sigma}_{i j 2}(0)+\left(k_{2}^{2} / k_{1}\right) \bar{r}_{c}^{0.276} \tilde{\sigma}_{i j 3}(0) \\
& +k_{4} \bar{r}_{c}^{0.341} \tilde{\sigma}_{i j 4}(0)+\left(k_{2}^{3} / k_{1}^{2}\right) \bar{r}_{c}^{0.497} \tilde{\sigma}_{i j 5}(0) .
\end{aligned}
$$

We take $J$ and $k_{2}$ as dominating parameters to develop a fracture criterion. $k_{4}$ is taken as a modified parameter. Using the last two relations, the lower bound toughness and upper bound toughness curve of $J_{c}-k_{2}$ are plotted in Fig. 10 for $\sigma_{c}=3.5 \sigma_{0}, J_{c}^{*}=40 \mathrm{Kpa.m}$ and $n=5$, corresponding to the maximum value and minimum values of $k_{4}$, respectively. The maximum and minimum values of $k_{4}$ corresponding to that for small scale yielding are $k_{4 \max }=0.1, k_{4 \min }=-0.1$. From Fig. 10 , it can be seen that the upper bound and lower bound of predicted toughness curves agree reasonably well with the experimental data.

\section{Concluding remarks}

A complete development for the higher-order asymptotic solution of the crack tip fields up to five terms for mode I loading of hardening materials in plane strain has been performed. At the same time, by numerical test, an additional conclusion is deduced, namely that in the higher-order asymptotic solution (to the twentieth order) for $n=3,5,10$, only three coefficients are independent. They are: the first term, second term, fourth term $(n=5,10)$ or fifth term $(n=3)$. By appropriate choices of the two coefficients, the higher-order asymptotic solutions accurately match the near tip fields generated by MBL analyses over the full range of loadings, and completely describe the near tip fields generated by finite element analyses over a wide range of crack geometries and external loadings.

Since only three parameters can be independently adjusted in the higher-order asymptotic solution, we consider establishing a modified $J-k_{2}$ two parameter fracture criterion based on the higher-order asymptotic solution. The third parameter $k_{4}$ or $k_{5}$ is taken as a modified term. The bound curves of $J-k_{2}$ are calculated when the third parameter is taken as its maximum value or minimum value in the critical state of crack initiation (here, $\sigma_{\theta \theta}=\sigma_{c}$, when $r=r_{c}, \theta=0$ ).

The upper bound and lower bound fracture toughness curves predicted by a modified two parameter criterion were given. These two curves agree well with the experimental data and fully capture their trend.

\section{References}

1. J.W. Hutchinson, Journal of the Mechanics and Physics of Solids 16 (1968) 13-31.

2. J.R. Rice and G.F. Rosengren, Journal of the Mechanics and Physics of Solids 16 (1968) 1-12.

3. J.R. Rice, Journal of Applied Mechanics 35 (1968) 379-386.

4. N.P. O'Dowd and C.F. Shih, in ASTM 24th National Symposium on Fracture Mechanics (1992).

5. A.M. Al-Ani and J.W. Hancock, Journal of the Mechanics and Physics of Solids 39 (1991) 23-43.

6. Y.C. Li and T.C. Wang, Scientia Sinica (Series A) 29 (1986) 941-955.

7. S.M. Sharma and N. Aravas, Journal of the Mechanics and Physics of Solids 39 (1991) 1043-1072.

8. S. Yang, Y.J. Chao and M.A. Sutton, Acta Mechanica 98 (1993) 79-98.

9. L. Xia, T.C. Wang and C.F. Shih, Journal of the Mechanics and Physics of Solids 41 (1993) 665-687.

10. N.P. O'Dowd and C.F. Shih, Journal of the Mechanics and Physics of Solids 39 (1991) 989.

11. Ibid, 40 (1992) 939.

12. C. Betegon and J.W. Hancock, Journal of Applied Mechanics 58 (1991) 104-110.

13. C.F. Shih and M.D. German, International Journal of Fracture 17 (1981) 27-43.

14. R.O. Ritchie, J.F. Knott and J.R. Rice, Journal of the Mechanics and Physics of Solids 21 (1973) 395-410.

15. M.T. Kirk, K.C. Koppenhoefer and C.F. Shih, ASTM STP (1991) in press. 